\title{
Influence of Processing Parameters on the Tensile Strength of PA-W/mLLDPE Composite Films Produced by Blown Film Extrusion
}

\author{
Nancy Zgheib ${ }^{(1)}$, Doumit Tahan ${ }^{(1)}$, Sylvain Seif ${ }^{(2)}$, Nemr El Hajj ${ }^{(1)}$ \\ (1) Department of Chemical and Mechanical Engineering, Faculty of Engineering, \\ Holy-Spirit University of Kaslik, P.O. Box 446, Jounieh, Lebanon \\ ${ }^{(2)}$ PACT R\&D Center, INDEVCO Group, Zouk Mosbeh, Lebanon
}

\begin{abstract}
Extrusion blown films from Polyamide waste/metallocene linear low-density polyethylene (PAW/mLLDPE) blend based matrix system and calcium carbonates particles were successfully produced and characterized. Also, the effect of the extruder temperature used during twin-screw compounding, the coupling agent loading and PA loading on the mechanical properties of the obtained films were studied. The key aspect of the present study was in investigating the optimal combination of factors by using Design of Experiment (DOE) approach. The responses measured were the tensile strength in the machine direction (MD) and the tensile strength in the transverse direction (TD). Among the selected processing parameters, the coupling agent and the interaction between the coupling agent and the PA loading have the most important influence on the tensile strength.
\end{abstract}

Keywords - PA-W/mLLDPE composites, blown film extrusion, Design of Experiment (DEO), tensile test

\section{INTRODUCTION}

Nowadays, plastic recycling is becoming an important challenge. The volumes of plastic wastes are increasing day by day, requiring effective recycling processes of different kinds of materials in a cost-efficient way [1]. Polyamide (PA) or nylon is one of the most common plastics used in food packaging. It is also used for medical and pharmaceutical packaging. Up to now, recycling of PA wastes (PA-W) is not well elaborated and few studies are available in the literature [2]. Several advances have been achieved in the plastic recycling technology; depolymerization by chemical recycling is a way to replay original monomers [3]. However, this method is difficult to achieve due to the complexity of systems and high cost of operation. The burning of polymers to recover energy is used too. In this case, environmental benefits are debatable [4]. Mechanical recycling is an interesting alternative which can combine technical viability, acceptable costs and environmental benefits. The presence of impurities and the degradation of the material are the most important obstacles for this method of polymer recycling $[5,6]$. In this work mechanical recycling was used to prepare polymer blends. PA-W/metallocene linear low-density polyethylene (mLLDPE) composite blends were produced using high-shear mixing twin screw extruder and the films were manufactured using a blown film extrusion process.
Within the scope of this study, a design of experiment (DOE) approach was investigated to determine the influence of many input variables on the output of the process used. Therefore, a series of structured tests were planned and the effects of the changes are then evaluated. A DOE study was made by Halim and al. [7] to investigate the influence of processing parameters on the mechanical properties of polyamide 11 composites. From this study, it was found that the nanoclay loading and the maximum barrel temperature are the significant variables that affect the mechanical properties of polyamide 11 composites. Furthermore, the influence of processing variables on the impact properties of the poly(butylene succinate) PBS/poly(butylene adipate-coterephthalate) PBAT/miscanthus biocomposites using a full factorial experimental design was studied by Mathuraj and al. [8]. The author demonstrates that the fiber length has the most important influence on the impact strength of the biocomposites. Another study by Johnson and al. [9, 10] showed that processing temperature affect largely the impact performance of Mater-Bi/miscanthus composites.

The aim of the present work is to optimize the fabrication of plastic films using PA-W/mLLDPE matrix with calcium carbonate as the reinforcing agent and varying several processing conditions (extruder temperature, coupling agent loading and PA loading). A full factorial design and analysis of variance (ANOVA) method was considered to analyze the effect of these parameters on the mechanical properties of the produced films. The effect of calcium carbonate loading level was not investigated in this work due to the fact that this loading level had already been optimized in a previous study. Besides, due to confidentiality agreements, applications of these plastic films are kept confidential.

\section{EXPERIMENTAL PROCEDURE}

\section{A. Materials}

The thermoplastic waste used in this work was PA-W from cast film extrusion obtained from INDEVCO, Lebanon. The PA-W is a copolymer $6 / 66$ grade of intermediate viscosity. The molecular weight of the $\mathrm{C}_{18} \mathrm{H}_{37} \mathrm{~N}_{3} \mathrm{O}_{5}$ monomer is 375.5 $\mathrm{g} / \mathrm{mol}$. The crystal structure of this PA-W is a shish-kebab type. The PE is a mLLDPE obtained from Exxon Mobil Chemical. The coupling agent is an anhydride-modified LLDPE resin obtained from DuPont Packaging \& Industrial 
Polymers. Calcium carbonates were obtained from Jordan Carbonate Company.

\section{B. Full factorial design}

A full factorial design was chosen in order to identify the influence of each variable as well as the interactions between the variables on the response. Using this statistical approach, there are $2 \mathrm{k}$ experiments, where ' $\mathrm{k}$ ' is the number of variables. In this study, the processing conditions being evaluated are coupling agent loading, PA loading and extruder temperature. As shown in TABLE I, two levels were assigned for each factor. MINITAB software was employed to make the experimental design and to analyze the obtained results through statistical plots at a confidence level of $95 \%$. For three independent variables there are eight experiments, each corresponding to a particular combination of factors as presented in TABLE II.

TABLE I. INDEPENDENT VARIABLES AND THEIR RESPECTED LEVELS

\begin{tabular}{|c|c|c|c|}
\hline Variables & Name & Lower level & Higher level \\
\hline 1 & $\begin{array}{c}\text { Extruder } \\
\text { temperature }\left({ }^{\circ} \mathrm{C}\right)\end{array}$ & 230 & 260 \\
\hline 2 & $\begin{array}{c}\text { Coupling agent } \\
\text { loading (\%) }\end{array}$ & 2.5 & 5 \\
\hline 3 & PA loading (\%) & 15 & 23.5 \\
\hline
\end{tabular}

\section{Melt compounding and blown film extrusion}

The production of the films is a two-stage process. The first stage is to produce a masterbatch by melt processing using a co-rotating twin screw extruder. At this stage, PA-W pellets are mixed with mLLDPE in the presence of the coupling agent and calcium carbonate filler to produce homogenized pellets or masterbatches. Before using the PA-W pellets, they were subjected to an overnight drying. The second stage of the film production is the processing of the masterbatches in a blown film extrusion. The film is pulled in the transverse direction (TD) to reach the ultimate diameter of the bubble. At the same time, it is being pulled in the machine direction (MD) by the movement of the downstream equipment. All twin screw compounding processes were carried out on a Leistritz Micro ZSE 27 HP twin screw extruder with a $27 \mathrm{~mm}$ screw diameter and a 48/1 length to diameter $(\mathrm{L} / \mathrm{D})$ ratio.

\section{Mechanical testing}

Tensile testing of plastic films was performed on an LLOYD universal testing machine using a $1 \mathrm{kN}$ load cell with a crosshead speed of $500 \mathrm{~mm} / \mathrm{min}$ in accordance with ASTM D882-12 at ambient temperature. A minimum of 5 test specimens were tested from each batch where the average data will be presented later in this paper. Tensile strength, tear resistance and dart drop impact tests were applied to the product to determine its mechanical properties. In this paper, we will present only the tensile test results.

\section{RESULTS AND DISCUSSION}

A DOE analysis was conducted to find out the effect of three factors on tensile strength of different blends. The objective of this study was to point out the most advantageous combination of factors (coupling agent loading, PA loading and extruder temperature). The results for the considered

TABLE II. DOE VARIABLES AND PA-W/MLLDPE COMPOSITE FORMULATIONS

\begin{tabular}{ccccc}
\hline Experiment & Extruder temperature $\left({ }^{\circ} \mathbf{C}\right)$ & Coupling agent loading (\%) & PA loading (\%) & Code \\
\hline 1 & 230 & 5 & 15 & 23.5 \\
2 & 230 & 2.5 & 23.5 & $230 \mathrm{~T} / 2.5 \mathrm{C} / 23.5 \mathrm{P}$ \\
3 & 260 & 2.5 & 15 & $260 \mathrm{~T} / 2.5 \mathrm{C} / 23.5 \mathrm{P}$ \\
4 & 260 & 5 & 15 & $260 \mathrm{~T} / 5 \mathrm{C} / 15 \mathrm{P}$ \\
5 & 230 & 2.5 & 15 & $230 \mathrm{~T} / 2.5 \mathrm{C} / 15 \mathrm{P}$ \\
6 & 260 & 2.5 & 23.5 & $260 \mathrm{~T} / 2.5 \mathrm{C} / 15 \mathrm{P}$ \\
7 & 230 & 5 & 23.5 & $23 \mathrm{~T} / 5 \mathrm{C} / 23.5 \mathrm{P}$ \\
8 & 260 & 5 & $\mathrm{~T} / 5 \mathrm{C} / 23.5 \mathrm{P}$ \\
\hline
\end{tabular}

TABLE III. MECHANICAL RESULTS FOR EACH BLEND

\begin{tabular}{cccc}
\hline Run & Code & Tensile Strength in MD (MPa) & Tensile Strength in TD (MPa) \\
\hline 1 & $230 \mathrm{~T} / 5 \mathrm{C} / 15 \mathrm{P}$ & $20.5 \pm 0.97$ & $16 \pm 1.6$ \\
2 & $230 \mathrm{~T} / 2.5 \mathrm{C} / 23.5 \mathrm{P}$ & $5.8 \pm 0.52$ & $5.3 \pm 0.17$ \\
3 & $260 \mathrm{~T} / 2.5 \mathrm{C} / 23.5 \mathrm{P}$ & $12.6 \pm 2.18$ & $9.1 \pm 0.67$ \\
4 & $260 \mathrm{~T} / 5 \mathrm{C} / 15 \mathrm{P}$ & $18.1 \pm 2.61$ & $16 \pm 1.31$ \\
5 & $230 \mathrm{~T} / 2.5 \mathrm{C} / 15 \mathrm{P}$ & $14.6 \pm 1.35$ & $13.3 \pm 2.01$ \\
6 & $260 \mathrm{~T} / 2.5 \mathrm{C} / 15 \mathrm{P}$ & $16.6 \pm 1.02$ & $16.2 \pm 0.95$ \\
7 & $230 \mathrm{~T} / 5 \mathrm{C} / 23.5 \mathrm{P}$ & $24.9 \pm 3.12$ & $19.9 \pm 1.71$ \\
8 & $260 \mathrm{~T} / 5 \mathrm{C} / 23.5 \mathrm{P}$ & $24.2 \pm 1.9$ & $20.3 \pm 0.47$ \\
\hline
\end{tabular}


responses (tensile strength in $\mathrm{MD}$ and $\mathrm{TD}$ directions) are shown in TABLE III. When comparing the results obtained for all the blends, it can be seen that the increase in the coupling agent loading from $2.5 \%$ to $5 \%$ increases the tensile stress in the machine direction and in the transverse direction for at least $40 \%$ for both configurations. Also, we can notice that an interaction exist between the coupling agent loading, the PA loading and the extruder temperature. This interaction will be studied further by the interaction plots and the analysis of variance (ANOVA).

\section{A. Pareto chart and Normal plot of the effects}

In order to visibly prove the impact of the independent variables on the mechanical properties of the different blends, the Pareto chart of effects is used. This chart shows the actual magnitude of each effect. Any factor that surpasses the reference line identified in red in the following chart is possibly important. Fig.1 shows the significant factors influencing the tensile strength in MD direction of the PAW/mLLDPE composite with a confidence level $(\alpha)$ of 0.05 . According to the Pareto chart, the coupling agent B had an important influence on the tensile strength of the produced films since the standardized effect value is greater than the reference line value 8.56.

Based on the normal plot of the effects, the points which appear far from the fitted line express a significant effect on the tensile strength. The points which are close to the fitted line show insignificant effects on the tensile strength. On the other hand, if the impact of the factor is negative, the concerning point will fall on the left side of the blue line. In Fig.1, it can be seen that the coupling agent is a significant factor since it is represented as square symbol and appears far away from the fitted line.

a)

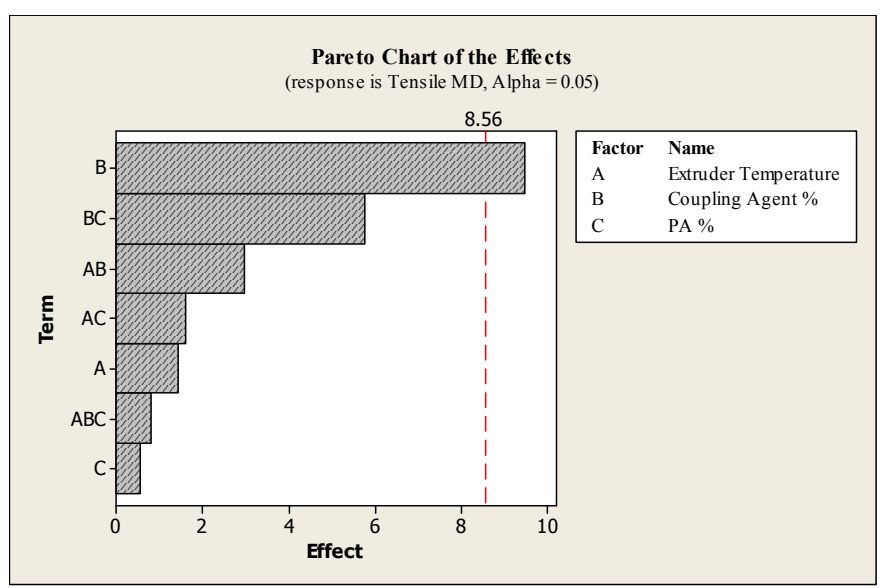

\section{B. Main effects plot}

It is possible to assess the effect of each parameter on the obtained results by using main effects plots. A main effect exists when the line connecting the mean values of the parameter is not horizontal. The sharper the slope of the line, the greater is the importance of the effect. On the other hand, if the line is parallel to the $\mathrm{x}$-axis, thus no main effect is present. Fig. 2 shows the main effects plot for the tensile strength in MD and TD directions of the resulting composite. Noticeably, coupling agent loading used during compounding has the greatest effect on the mechanical properties of the blends which is illustrated with a strong line slope. On the other hand, the extruder temperature and PA loading have a slight effect on the tensile strength in TD and MD directions. Also, PA loading shows a negative effect on the response when varying its levels.

\section{Interaction Plot and Analysis of Variance (ANOVA)}

In order to evaluate the interaction of two factors or more on the obtained results and examine their strength, the interaction plot is utilized. If the lines drawn by the software are not parallel, there is possibility for an interaction between the concerned factors and vice-versa.

ANOVA can be used to study the effects of factors with respect to response. The individual and interaction factors influence on the mechanical properties are estimated by using the sum of square (SS), mean square (MS), F-test statistics and P-values. In order to evaluate the effect of the chosen factors on the factorial design experiment, a 95\% confidence interval was selected with an alpha level $(\alpha)=0.05$. Usually, if the Pvalue is less than 0.05 , the considered factor has significantly affected the experiment response.

b)

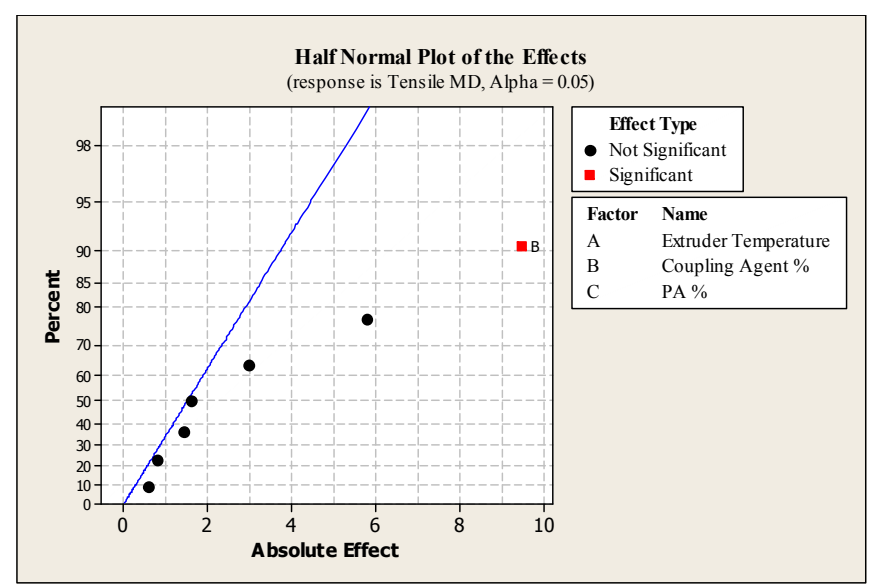

Fig. 1. Represents a) the Pareto chart and b) the Half normal plot of the effects for the tensile strengh in MD direction of the PA-W/mLLDPE composite 
a)

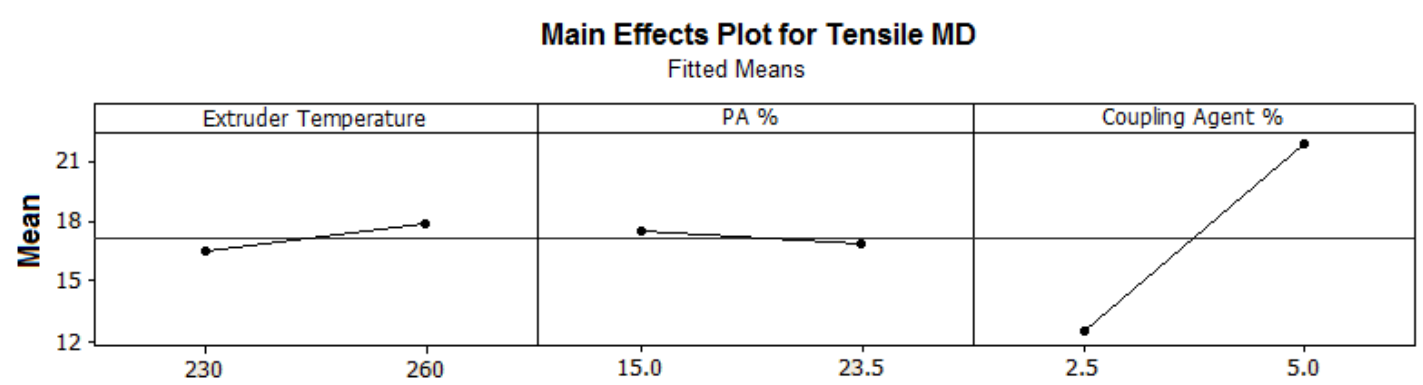

b)

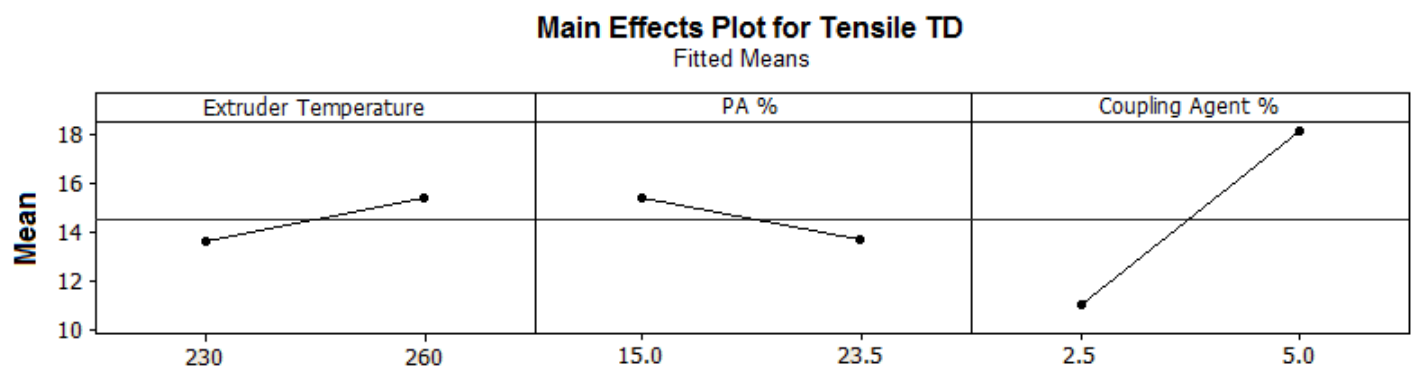

Fig. 2. Represents the main effects plot for tensile strength in a) MD and b) TD directions of the PA-W/mLLDPE composite

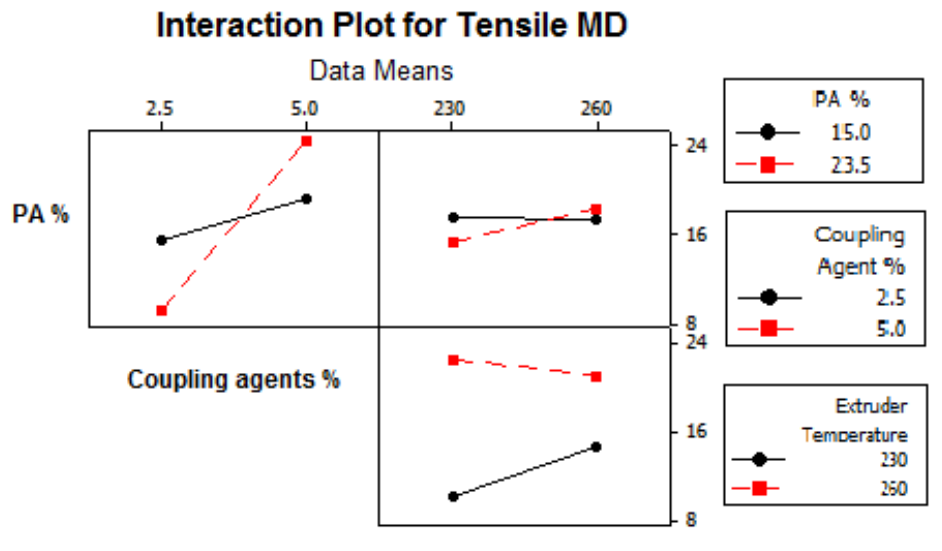

Extruder Temperature

Fig. 3. Interaction plot for tensile strength in MD direction of the PA-W/mLLDPE composite

Fig.3 represents the statistically significant binary interaction effect between the selected variables on the tensile strength in MD direction. Clearly, the PA loading/coupling agent loading, PA loading/extruder temperature and coupling agent loading/extruder temperature have an interaction. Out of the selected variables the combination PA loading/coupling agent loading had the most significant effect on tensile strength while the combination coupling agent loading/extruder temperature had the least significant effect. These results are confirmed by the analysis of variance illustrated in TABLE IV when the P-values between the different combinations are compared. Fig.4 illustrates the interaction plots for the tensile strength in TD direction of the composite. The three combinations show a possible interaction. It can be noticed that the most significant interaction among the different parameters is the combination PA loading/coupling agent loading. The analysis of variance results illustrated in TABLE $\mathrm{V}$ shows that the three chosen variables for this study have an impact on the tensile strength in the TD direction. PA loading, coupling agent loading and extruder temperature have respectively a P-value of $0.041,0.01$ and 0.04 . According to these results the coupling agent loading has the lowest P-value; consequently it is the most influencing factor for this property. The combination PA loading/coupling agent loading and coupling agent loading/extruder temperature have also an effect on the tensile strength in TD direction. 
TABLE IV. ANAlysis of VARIANCE For TENSILE StRENGTH IN MD DiRECTION OF THE PA-W/MLLDPE COMPOSITE

\begin{tabular}{lccccc}
\hline \multicolumn{1}{c}{ Source } & DF & Sum of squares (SS) & Mean squares (MS) & F & P \\
\hline PA \% & 1 & 0.657 & 0.657 & 0.52 & 0.601 \\
Coupling agent \% & 1 & 180.273 & 180.273 & 143.64 & 0.053 \\
Extruder temperature & 1 & 4.042 & 4.042 & 3.22 & 0.324 \\
PA \%*Coupling agent \% & 1 & 66.809 & 66.809 & 53.23 & 0.087 \\
PA \%*Extruder temperature & 1 & 5.184 & 5.184 & 4.13 & 0.291 \\
Coupling agent \% * Extruder temperature & 1 & 17.754 & 17.754 & 14.15 & 0.165 \\
Error & 1 & 1.255 & 1.255 & \\
Total & 7 & 275.974 & & & \\
S=1.12027 R-SQ=99.55\% R-SQ(ADJ) =96.82\% & & & &
\end{tabular}

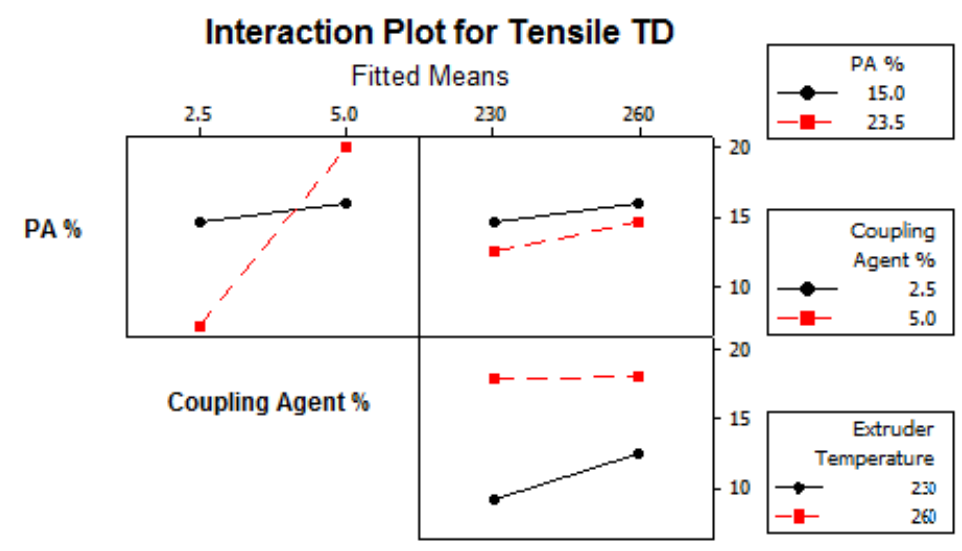

Extruder Temperature

Fig. 4. Interaction plot for Tensile Strength in TD direction of the PA-W/mLLDPE composite

TABLE V. ANALYSIS OF VARIANCE FOR TENSILE STRENGTH IN TD DIRECTION OF THE PA-W/MLLDPE COMPOSITE

\begin{tabular}{|c|c|c|c|c|c|}
\hline Source & DF & Sum of squares (SS) & Mean squares (MS) & $\mathbf{F}$ & $\mathbf{P}$ \\
\hline PA $\%$ & 1 & 6.016 & 6.016 & 239.66 & 0.041 \\
\hline Coupling Agent \% & 1 & 100.74 & 100.74 & 4013.46 & 0.01 \\
\hline Extruder Temperature & 1 & 6.431 & 6.431 & 256.19 & 0.04 \\
\hline PA $\% *$ Coupling agent $\%$ & 1 & 67.507 & 67.507 & 2689.46 & 0.012 \\
\hline PA $\% *$ Extruder temperature & 1 & 0.225 & 0.225 & 8.96 & 0.205 \\
\hline Coupling agent $\% *$ Extruder Temperature & 1 & 5.097 & 5.097 & 203.07 & 0.045 \\
\hline Error & 1 & 0.025 & 0.025 & & \\
\hline Total & 7 & 186.041 & & & \\
\hline
\end{tabular}

The low P-value (0.012) for the interaction of PA loading/coupling agent loading in addition to the interaction plot, confirm that this combination is the worth mentioning one.

\section{CONCLUSION}

PA-W/mLLDPE composite films have been successfully produced and characterized in this study. The influence of the independent processing variables on the tensile strength of the produced films in machine direction and transverse direction has been investigated by $2^{3}$ full factorial design of experiment. The statistically significant main and interaction variables were analyzed at a 95\% confidence level. According to main effect plot, Pareto plot, and half normal plot, the coupling agent loading plays an important role on the tensile strength of these films. On the other hand, the interaction plot and the analysis of variance show that the binary interaction between PA loading/coupling agent loading has the most significant response among the selected variable combinations. This result proves that the maleic anhydride is a suitable coupling agent for compatibilizing normally incompatible polymers like PA and mLLDPE. For future work, we can choose more number of parameters such as the particle size of calcium carbonate filler or the screw speed in order to get the optimum mechanical properties. 


\section{ACKNOWLEDGMENT}

This work has been funded by INDEVCO PACT (Polymer Application Center for Technology) R\&D Center. We give special thanks to Dr. Sylvain Seif and Dr. Johnny Najem of PACT for their support and fruitful scientific discussions.

\section{REFERENCES}

[1] B. Acevedo, A.M. Fernández, C. Barriocanal, Identification of polymers in waste tyre reinforcing fiber by thermal analysis and pyrolysis, Journal of Analytical and Applied Pyrolysis, 111 (2015) 224-232.

[2] E. Silva, Recycled polyamides, a literature review and research opportunities, College of Textiles, NCSU, 2008: 2-7, 14.

[3] H. Nishida, Development of materials and technologies for control of polymer recycling, Polym. J. 43 (2011) 435-447.

[4] S.M. Al-Salem, P. Lettieri, J. Baeyens, The valorization of plastic solid waste (PSW) by primary to quaternary routes: from re-use to energy and chemicals, Prog. Energy Combust, 36 (2010) 103-129.
[5] R. Laryea-Goldsmith, J. Oakey, N.J. Simms, Gaseous emissions during concurrent combustion of biomass and no-recyclable municipal solid waste, Chem. Cent. J. 5 (2011) 2-10

[6] C T. Ferreira, C. A.B. Perez, D. Hirayama, C.Saron: Recycling of polyamide (PA) from scrap tires as composites and blends, Journal of Environmental Chemical Engineering, 1 (2013) 762-767.

[7] K. A. Abdul Halim, J. B. Farrell, J.E. Kennedy, Optimisation of a twin screw extrusion process for enhanced short-term mechanical properties of polyamide 11 nanocomposites using Design of Experiment (DOE) approach, Conference paper, June 2012.

[8] R. Muthuraj, M. Misra, F. Defersha, A. Mohanty, Influence of processing parameters on the impact strength of biocomposites: A statistical approach, Composites: Part A 83 (2016) 120-129.

[9] R.M. Johnson, N. Tucker, S. Barnes, Impact performance of miscanthus/NovamontMater-Bi biocomposites, Polym Test 2003, 22 (2): 209-15.

[10] M. Johnson, N. Tucker, S. Barnes, K. Kirwan, Improvement of the impact performance of a starch based biopolymer via the incorporation of Miscanthus giganteus fibres, Ind Crops Prod 2005, 22 (3):175-86. 